\title{
Using Fluorescence Lymphangiography to Define the Ileocolic Mesentery: Proof of Concept for the Watershed Area Using Real-time Imaging
}

Deborah S Keller, MS MD, Department of Surgery, University College London Hospital, London, UK; Division of Colorectal Surgery, Department of Surgery, Baylor University Medical Center, Dallas, TX, USA

Heman M Joshi, MBChB, MRCS (Eng) Department of Surgery, University College London Hospital, London, UK

Manuel Rodriguez-Justo, MD, Department of Histopathology, University College London Hospital, London, UK

Dara Walsh, Centre for Interventions in Infection, Inflammation and Immunity, Graduate Entry Medical School, University of Limerick, Limerick, Ireland.

John Calvin Coffey, Department of Surgery, University Hospital Limerick, Limerick, Ireland

Manish Chand, MBBS MBA FRCS PhD, Department of Surgery, University College London Hospital, London, UK

Word Count: 947

Corresponding Author:

Manish Chand, MBBS MBA FRCS PhD

Consultant Colorectal Surgeon \& Senior Lecturer

Department of Surgery, University College London Hospital

250 Euston Rd, Bloomsbury, London NW1 2BU

Tel: 02034475879

Fax: 02034479218

manish.chand@uclh.nhs.uk 


\title{
Technical Note
}

\begin{abstract}
Recent advances in mesenteric science have demonstrated that the mesentery is a continuous structure with a 'watershed' area at the mesenteric apex between the right colon and terminal ileum, where lymphatic flow can proceed either proximally or distally. With this new understanding of the anatomy, functional features are emerging, which can have an impact on surgical management. Fluorescence lymphangiography or lymphoscintigraphy with indocyanine green (ICG) allows real-time visualization of lymphatic channels, which highlights sentinel lymph nodes and may facilitate identification of the ideal margins for mesenteric lymphadenectomy during bowel resection for colon cancer. By using this novel technology, it is possible to demonstrate a watershed area in the ileocolic region, and may facilitate more precise mesenteric dissection. In the present study we provide proof of concept for the ileocolic watershed area using fluorescence lymphangiography.
\end{abstract}

Keywords:Ileocolic; Mesentery; Watershed area; Fluorescence lymphangiography;Oncologic resection.

\section{Introduction}

A watershed area is classically described as a region that receives a dual blood supply from the most distal branches of two large arteries. Recent advances in anatomical science have recognized the mesentery as an organ (1). In this model, the right mesocolon is continuous with the small intestinal mesentery. As a result of this continuity, there is an 
apex with a substantial lymphatic mass in the ileocecal region, the confluence between the terminal ileum and right mesocolic mesentery, and a lymphatic watershed area is created. Here, the lymphatic drainage can proceed proximally, up along the small intestinal region of mesentery, or distally, along the right mesocolic mesentery $(1,2)$ (Fig. 1). With this new understanding of the anatomy, functional features are emerging which could guide surgical dissection, especially in colorectal cancer patients (1).

The principles of oncologic resection for colorectal cancer are based on excising the primary tumor with its blood supply and lymph node basin. In rectal cancer, embryological fascial planes define the resection bounds, and total mesorectal excision (TME) is the standard of care $(3,4)$. In colon cancer, the magnitude of the mesocolic excision and need for a complete mesocolic excision (CME) is controversial. Proponents of CME argue that performing more radical surgery with central vascular ligation and a larger mesenteric specimen results in a greater number of lymph nodes, an oncologically superior specimen, and potentially improved survival outcomes $(5,6)$. However, opponents have demonstrated that wider excision in right-sided hemicolectomies for colon cancer have no oncological benefit, and voice concerns about increased morbidity after extended lymph node dissection, increased postoperative mortality, and a decreased 5-year overall survival (79). Thus, there is no current consensus for a CME approach.

An alternative to this radical resection could be a more precise resection using fluorescence lymphangiography or lymphoscintigraphy with indocyanine green (ICG). The ICG-based approach allows real-time visualization of lymphatic channels and highlights sentinel 
lymph nodes (10-12). This technology could theoretically delineate the precise lymphatic drainage within the mesentery, thereby identifying the ideal margins for mesenteric lymphadenectomy during bowel resection for colon cancer. By using this novel technology, it is possible to demonstrate the principles of a watershed area in the ileocolic region, which challenges the traditional anatomical teaching, and guide accurate mesenteric resection.

Inthe present study, we provide proof of concept for the ileocolic watershed area.

\section{Materials and methods}

We present the case of a 73-year-old female who underwent a colonoscopy for obstructive symptoms, and was found to have a mass in the proximal ascending colon occupying $2 / 3$ of the lumen. The lesion was tattooed to aid intraoperative identification. The pathology for the colonoscopy biopsies was moderately differential carcinoma. On a staging computed tomography scan of the abdomen the mass was identified as T2N1 ascending colon cancer. Therefore, the patient underwent an elective laparoscopic right hemicolectomy. The lesion was not clearly visible on laparoscopic exploration despite the tattoo. To ensure an appropriate oncologic resection was performed, we sought to visualize the mesentery involved with the tumor, its blood flow and lymphatic map, before determining the resection margins. A standard medial-to-lateral mobilization was performed, with intracorporeal ileocolic artery ligation; then, the specimen was extracorporealized. There was no intracorporeal mesenteric division. A $1 \mathrm{~mL}$ volume of ICG (concentration $5 \mathrm{mg} / 10 \mathrm{~mL}$ ) was injected into the subserosal layer of the bowel around the tumor in 4 sites. Using a laparoscope from a commercially-available fluorescence 
imaging system with near infrared (NIR) function we visualized the ileocolic watershed area, a line of lymphatics traveling across the mesentery, as it was excited by fluorescent light in the NIR spectrum in real-time (Fig. 2). Though visualization occurred within 60 seconds, we waited a total of 5 minutes to ensure full effect.

\section{Results}

Based on the fluorescence lymphangiography and (separately performed) angiography, the precise amount of mesentery and bowel was resected to ensure adequate margins and perfusion. The final pathology on the mass revealed an R0 resection of a T3N0 adenocarcinoma, with 35 lymph nodes harvested, and 0 involved (Fig. 3). There were no adverse events or injection-related issues. The patient's postoperative course was uneventful, and she was discharged to home on postoperative day 4.

\section{Discussion}

Fluorescence imaging has been shown to effectively and objectively demonstrate perfusion in the gastrointestinal tract (13). More novel uses of ICG have been recently described including identification of sentinel nodes and lymphatics (10). Prior studies have shown the efficacy and ability to visualize the mesenteric and central vessels for ligation $(14,15)$. We demonstrated that the technology can be applied to direct more precise surgery, especially in right colon cancers, guiding an optimal oncological resection and avoiding the need for $\mathrm{CME}$ and its associated risks. As new anatomical concepts emerge, there is an opportunity to reassess how we approach oncological resections in colon cancer and to optimize nodal yields and improve survival outcomes. 
To our knowledge this is the first demonstration of the watershed concept using fluorescence imaging technology. With ICG fluorescence lymphangiography, a clear demarcation was seen in the ileocolic mesentery, establishing the ileocolic lymphatic watershed area. This demarcation can be used to determine the appropriate mesenteric lymphadenectomy. There are clinical implications for using these boundaries to guide optimal oncological resection, similar to the concept of the "holy plane" for TME $(3,4$, 16). ICG fluorescence lymphangiography of the watershed area could prevent unnecessary resection of uninvolved mesentery and ensure that the precise mesenteric regions involved in lymphatic drainage are resected. In addition, there are implications for teaching the new understanding of the mesentery, using 3-D virtual modeling and ICG fluorescence lymphangiography. Further study is needed to validate the concept of intraoperative fluorescence lymphangiography.

Conflict of interest: the authors declare that they have no conflict of interest 


\section{Figure Legends}

Figure 1a- Contemporary model of the right colon mesentery as a continuous and contiguous structure; $1 \mathrm{~b}$. The same view of the mesentery is seen real-time after ICG injection. Here, the ileocolic watershed area is indicated by the faint line of lymphatics travelling along the colonic mesentery (red arrows). This line corresponds to the red line in figure 1a. Photo a courtesy of John Calvin Coffey

Figure 2- Appearance of ICG in tumor (black arrows) and the sentinel lymphatics. The ileocolic watershed area is demonstrated by the faint line of lymphatics travelling along the colonic mesentery (red arrows).

Figure 3- Pathologic specimen for the R0 resection of a T3N0 adenocarcinoma, with 35 lymph nodes harvested. 


\section{References}

1. Coffey JC, O'Leary DP. (2016)The mesentery: structure, function, and role in disease. Lancet Gastroenterol Hepatol. ;1:238-247.

2. Coffey JC, O'leary DP.(2017) Defining the mesentery as an organ and what this means for understanding its roles in digestive disorders. Expert Rev Gastroenterol Hepatol. . 11(8):703-705

3. Heald RJ, Husband EM, Ryall RD. (1982)The mesorectum in rectal cancer surgery-the clue to pelvic recurrence? Br J Surg. ;69:613-616.

4. Heald RJ.(1988) The 'Holy Plane' of rectal surgery. J R Soc Med. ;81:503-508.

5. Hohenberger W, Weber K, Matzel K, Papadopoulos T, Merkel S.(2009) Standardized surgery for colonic cancer: complete mesocolic excision and central ligation--technical notes and outcome. Colorectal Dis. ;11:354-64; discussion 364.

6. West NP, Hohenberger W, Weber K, Perrakis A, Finan PJ, Quirke P.(2010) Complete mesocolic excision with central vascular ligation produces an oncologically superior specimen compared with standard surgery for carcinoma of the colon. J Clin Oncol. ;28:272-278.

7. Olofsson F, Buchwald P, Elmståhl S, Syk I. (2013)Wide excision in right-sided colon cancer is associated with decreased survival. Scand J Surg.;102:241-245.

8. Olofsson F, Buchwald P, Elmståhl S, Syk I.(2016) No benefit of extended mesenteric resection with central vascular ligation in right-sided colon cancer. Colorectal Dis. ; 18:773-778.

9. Bertelsen CA, Kirkegaard-Klitbo A, Nielsen M, Leotta SM, Daisuke F, Gögenur I.(2016) Pattern of Colon Cancer Lymph Node Metastases in Patients Undergoing Central Mesocolic Lymph Node Excision: A Systematic Review. Dis Colon Rectum. ;59:1209-1221.

10. Cahill RA, Anderson M, Wang LM, Lindsey I, Cunningham C, Mortensen NJ(2012). Near-infrared (NIR) laparoscopy for intraoperative lymphatic roadmapping and sentinel node identification during definitive surgical resection of early-stage colorectal neoplasia. Surg Endosc. ;26:197-204.

11. Verbeek FP, Tummers QR, Rietbergen DD et al.(2015) Sentinel Lymph Node Biopsy in Vulvar Cancer Using Combined Radioactive and Fluorescence Guidance. Int J Gynecol Cancer. ;25:1086-1093.

12. Kusano M, Tajima Y, Yamazaki K, Kato M, Watanabe M, Miwa M.(2008) Sentinel node mapping guided by indocyanine green fluorescence imaging: a new method for sentinel node navigation surgery in gastrointestinal cancer. Dig Surg. ;25:103108.

13. Jafari MD, Wexner SD, Martz JE et al.(2015) Perfusion assessment in laparoscopic left-sided/anterior resection (PILLAR II): a multi-institutional study. J Am Coll Surg. ;220:82-92.e1.

14. Watanabe J, Ota M, Suwa Y, Ishibe A, Masui H, Nagahori K(2016). Real-Time Indocyanine Green Fluorescence Imaging-Guided Complete Mesocolic Excision in Laparoscopic Flexural Colon Cancer Surgery. Dis Colon Rectum. ;59:701-705.

15. Watanabe J, Ota M, Suwa Y, Ishibe A, Masui H, Nagahori K.(2017) Evaluation of lymph flow patterns in splenic flexural colon cancers using laparoscopic real-time indocyanine green fluorescence imaging. Int J Colorectal Dis. ;32:201-207.

16. Kraima AC, West NP, Treanor D et al. (2015) Understanding the surgical pitfalls in 
total mesorectal excision: Investigating the histology of the perirectal fascia and the pelvic autonomic nerves. Eur J Surg Oncol. ;41:1621-1629. 